\title{
Next Generation Sequencing and Its Clinical Applications: The Growing Pains
}

\section{Ken CN Chang ${ }^{1^{*}}$ and Matthew J Marton ${ }^{2}$}

${ }^{1}$ Translational Molecular Biomarkers, Merck Research Laboratories, Merck \& amp, Co., Inc., 126 E. Lincoln Avenue, Rahway, NJ 07065, USA

${ }^{2}$ Companion Diagnostics, Translational Biomarkers, Merck Research Laboratories, Merck \& amp, Co., Inc., 126 E. Lincoln Avenue, Rahway, NJ 07065, USA

"Corresponding author: Ken CN Chang, Translational Molecular Biomarkers, Merck Research Laboratories, Merck \& amp, Co., Inc., 126 E. Lincoln Avenue, Rahway, NJ 07065, USA, Tel: +19084231000; E-mail: ken.chang@merck.com

Rec date: Jul 28, 2016; Acc date: Aug 27, 2016; Pub date: Aug 29, 2016

Copyright: (c) 2016 Chang K, et al. This is an open-access article distributed under the terms of the Creative Commons Attribution License, which permits unrestricted use, distribution, and reproduction in any medium, provided the original author and source are credited.

\section{Short Communication}

As the list of applications of next generation sequencing (NGS)based assays continues to grow and the user network continues to expand from academic and pharmaceutical discovery research to clinical decision-making tests, the challenges and controversies continue to persist. Over the past year, the FDA held several workshops on the analytical and clinical validation of NGS tests that resulted in the release of two guidance documents, for which it has requested feedback from NGS stakeholders [1,2]. One key area of debate is defining the best method for establishing analytical validity of NGS tests (use of standards or the use of processes such as quality system regulations, QSRs). Some feel that QSRs are overly burdensome, impractical and cost-prohibitive; others feel the use of standards may be insufficiently rigorous. Our concern is how the standards would be implemented. Another question is related to whether or how clinical labs should confirm novel variant calls. For example, if a whole exome sequencing (WES) of a sample results in 500 somatic mutation calls, it would be essentially impossible (both cost prohibitive and time-consuming) to confirm all 500 variants using an orthogonal method. In some clinical or hospital settings, verifying specific decision-making variant calls for a given patient with special disease condition using orthogonal methods might be feasible or justifiable. However, if the assay is to be used for the determination of patients' hyper-mutation status within a clinical trial, it might be unrealistic to confirm every potential variant call.

A third controversy is whether clinical databases could be used for clinical validation of novel mutations not in the literature, especially those mutations for which analytical confirmation was not explicitly performed. Unfortunately, if a public database of genotype-phenotype associations were created using historic data with limited variant confirmation or reproducibility measures in place, even if the data were from reputable labs, using such a database to support clinical validity of an NGS-based in vitro diagnostic might have unintended consequences, and could even increase the risk of getting incorrect diagnoses in the clinic $[3,4]$. Of course, if the database only contained those specific variants directly supported by the clinical evidence of genotype-phenotype association on a variant-by-variant basis, then that might be acceptable and useful. We can illustrate our concern with an extreme example: suppose a patient's sample that has 500 variants derived from its WES data is confirmed to be a responder for a given treatment, are we saying that now all 500 variants are considered validated and should be deposited into this public database? We would argue against this for several reasons, not the least of which would be the lack of confirmation of each mutation.
As for the use of standards in NGS-based in vitro diagnostics for germline diseases, although it should have substantial value in terms of serving as control samples to ensure basic quality of procedures are met, similar concerns exist. Analyzing a standard a single time may be an ineffective way to assess the analytical performance of a given method, at least for less common mutation sites. Our lab investigated mutation call reproducibility by analyzing formalin fixed and paraffin embedded (FFPE) standards (i.e. control samples) using triplicate library preparations. While we found hotspot mutations were called consistently, the same was not true for non-hotspot locations since many non-hotspot variant calls generated from individual testing FFPE were not reproducible. In addition, among the reproducible variant calls some were platform or panel-specific artifacts that showed up across all samples including reference samples (Chang et al. manuscript to be submitted).

Our recent publication revealed the highly fragmented nature of DNA in FFPE tissue samples and the subsequent amplification of the short fragments during library preparation are responsible for many false positive variant calls [5]. These false positive calls are not due to result from a lack of repeatability in the sequencing since most current widely-accepted NGS technologies permit excellent instrument run-torun repeatability if same library preparation is used [5]. Instead, the genesis of the false positive calls lies in the library preparation step. For example, when different library preparations are involved, even starting with same DNA or RNA stock solution, the reproducibility of potential somatic mutation calls (whose variant frequencies usually fall below 25\%) is in general poor [5]. For WES this seems particularly concerning, considering the work of Belkadi et al. who showed that more than $50 \%$ of high quality variant calls were false positives by using Sanger sequencing to confirm randomly selected single nucleotide variant calls [6]. Our un-published studies are in agreement with this finding as mutation calls from three library preparations from the same batch of FFPE tissue DNA showed less than $50 \%$ of all variant calls generated from WES were shared by the three replicates even though high quality filters were applied (Chang et al. manuscript in preparation). Our results also indicate that using independent library preparation replicates is an effective way to identify false positive calls [5]. Recently, O'Rawe et al. showed that NGS analysis of the same data set using different variant caller pipelines often resulted in low concordance [7]. Even though restricting ones focus to only the shared variant calls from multiple data analysis pipelines may be an effective way to eliminate some false positives, this approach will not be able to eliminate certain artifacts as effectively as the triplicate approach. For example, use of multiple pipelines is unlikely to eliminate artifact variants created during or after the tissue collection, such as those resulting from deamination or oxidation; whereas we have clearly 
Citation: Chang KCN, Marton MJ (2016) Next Generation Sequencing and Its Clinical Applications: The Growing Pains. Next Generat Sequenc

Page 2 of 2

shown that the replicate approach can essentially eliminate this class of artifacts.

How do we take advantage of the improved accuracy achieved by performing replicates while minimizing the cost and effort associated with replicates? Since most people agree that the routine running of replicates to verify each and every variant call reported from a clinical NGS assay is impractical and cost prohibitive, a compromise is needed. We propose the following concept: for clinical lab to include at least one unique randomly selected clinical sample from a previously run in each and every new batch of NGS clinical assay/test, starting from nucleic acid. Therefore, over a period of time and through the entire sample testing process, some degree of real-world intra-laboratory reproducibility of clinical samples will be available in terms of the total number and the identity of mutations reported. Further discussion of this concept will be submitted for publication in the near future.

\section{References}

1. Use of Public Human Genetic Variant Databases to Support Clinical Validity for Next Generation Sequencing-Based in Vitro Diagnostics (2016) FDA guidance document- July 2016 [http://www.fda.gov/ucm/ groups/fdagov-public/@fdagov-meddev-gen/documents/document/ ucm509837.pdf] Center for Biologics Evaluation and Research. Accessed on: August 27, 2016.
2. Use of Standards in the Food and Drug Administration's Regulatory Oversight of Next Generation Sequencing-Based in Vitro Diagnostics Used for Diagnosing Germline Diseases (2016). FDA guidance document- July 2016 [http://www.fda.gov/ucm/groups/fdagov-public/ @fdagov-meddev-gen/documents/document/ucm509838.pdf] Center for Biologics Evaluation and Research. Accessed on: August 27, 2016.

3. Gerlinger M, Rowan AJ, Horswell S, Larkin J, Endesfelder D, et al. (2012) Intratumor Heterogeneity and Branched Evolution Revealed by Multiregion Sequencing. New England J Med 366: 883-892.

4. Alexandrov LB, Nik-Zainal S, Wedge DC, Aparicio SA, Behjati S, et al. (2013) Signatures of mutational processes in human cancer. Nature 500: 415-421.

5. Chang KCN, Zhao Y, Kang J, Pant S, Qiu P, et al. (2014) Validation of Next Generation Sequencing Cancer Panels for Clinical Somatic Mutation Profiling: Identification of Source of Variations and Artifacts using FFPE Tissues. Journal of Next Generation: Sequencing and Applications (JNGSA) 1: 109.

6. Belkadi A, Bolze A, Itan Y, Cobat A, Vincent Q, et al. (2015) Wholegenome sequencing is more powerful than whole-exome sequencing for detecting exome variants. PNAS 112: 5473-5478.

7. O'Rawe J, Jiang T, Sun G, Wu Y, Wang W, et al. (2013) Low concordance of multiple variant-calling pipelines: practical implications for exome and genome sequencing. Genome Medicine 5: 28. 